\title{
Solar Energy and Nanomaterials for Clean Energy Development
}

\author{
M. S. A. Abdel-Mottaleb, ${ }^{1}$ Frank Nüesch, ${ }^{2}$ and Mohamed M. S. A. Abdel-Mottaleb ${ }^{3}$ \\ ${ }^{1}$ Nano-Photochemistry and Solarchemistry Laboratory, Department of Chemistry, Faculty of Science, \\ Ain Shams University, Abbassia, Cairo 11566, Egypt \\ ${ }^{2}$ Laboratory for Functional Polymers, EMPA, Überlandstrasse 129, CH-8600 Dübendorf, Switzerland \\ ${ }^{3}$ Nanotechnology Program, Nile University, Smart Village-B71 K28, Cairo/Alex Desert Road, Cairo, Egypt \\ Correspondence should be addressed to M. S. A. Abdel-Mottaleb, phochem08@photoenergy.org
}

Received 31 December 2009; Accepted 31 December 2009

Copyright (C) 2009 M. S. A. Abdel-Mottaleb et al. This is an open access article distributed under the Creative Commons Attribution License, which permits unrestricted use, distribution, and reproduction in any medium, provided the original work is properly cited.

This special issue on "Solar energy and nanomaterials for clean energy development" is composed of selected, fulllength versions of papers presented during the international Solar'09 conference that was held in the fascinating historical city of Luxor. The conference gathered scientists from 26 countries, to discuss outstanding research on a multitude of topics and disciplines. As was pointed out by Professor Paul Barbara from the University of Texas in Austin at the opening session of the conference, this medium-sized conference offered the unique opportunity to learn and exchange scientific issues from distinct disciplines that have one main thing in common, solar photons. This exceptional opportunity to learn about other fields of research not only required particular didactic skills form the speakers, but also demanded special attention and openness from the audience.

Under the headline of "powering a greener future," a total of 41 oral presentations were delivered and complemented by a similar number of posters. Photovoltaics, photocatalysis, solar irradiance, photodegradation, photoinduced electron transfer, photochemistry, photobatteries and photospectroscopy are just a flavour of the high diversity of discussed topics. There was even an enriching contribution on pellet fuels for future generation IV nuclear plants, reminding that continued progress is also necessary in already proven technologies. A strong emphasis was placed on organic-based photovoltaics. Dye-sensitized solar cells have now reached a high degree of maturity in theoretical and experimental understanding of the various microscopic processes contributing to the overall working principle. The dye-sensitized (Grätzel) solar cell is the earliest solar cell directly utilizing nanoscale components for its performance. Here, light absorption takes place predominantly in dye molecules anchored to the surface of nanoparticles of a wide bandgap semiconductor, usually $\mathrm{TiO}_{2}$. Upon excitation with visible light, the dye injects electrons from its excited state into the $\mathrm{TiO}_{2}$ conduction band, leading to a charge-separated state. To absorb enough light, the $\mathrm{TiO}_{2}$ is made nanoporous, with a large surface area per unit volume and weight. The optimization of the dye/ $/ \mathrm{TiO}_{2}$ layer is heavily dependent on nanoscale fabrication and characterization; the $\mathrm{TiO}_{2}$ layer must have a large surface area to incorporate enough dye, but a structure that is thin and open enough to allow for efficient charge transport with minimum losses. Above all, the quest for novel dyes in dye sensitized solar cells is clear, for efficient near-infrared absorbers that are still missing. Interfacial charge transfer complexes on $\mathrm{TiO}_{2}$ showed a new way to achieve direct interfacial charge-transfer. Due to the important advances worldwide, this thin film photovoltaic technology is on the verge of large-scale commercialisation. In solid organic solar cells, higher efficiencies have still to be met. Fundamental studies are certainly required for this technology to be in accordance with dye-sensitized solar cells. As was shown in one contribution, single molecule fluorescence spectroscopy can reveal microscopic response of conjugated polymers to charging. Single-molecule probes were also shown to be new experimental tools to explore nanoporous 
materials, in addition to examining light-harvesting systems. Classical photographic dyes have also been proposed as new material class for organic solar cells. Novel device concepts based on plasmon resonances in nanoparticles were proposed.

Besides photovoltaics, photocatalysis was another theme encompassing the four conference days. Photocatalized decomposition of pollutants at various titania surfaces is of great interest. Electron transfer reactions are at the base of these photoinduced processes and were also the subject of one of the theoretical contributions. Water purification, pollutant degradation, and hydrogen production were the most targeted applications. The principle of photocatalysis is to use the energy of photons in the visible to near-UV range (ca. 1.5-5 eV) to generate active chemical species driving a surface chemical reaction on a photocatalyst.

The typical scheme of photocatalysis involves harvesting of light photons in a semiconductor (most commonly $\mathrm{TiO}_{2}$ ), and subsequent conversion of these photons to electronic excitations, which then induce the desired chemical reaction on the semiconductor surface. One main bottleneck of this scheme is a high recombination rate of the excited electronhole pairs, which is relatively slower in case of semiconductor particles of nanodimensions. The majority of charge carriers in nanoparticles will have the opportunity of diffusing into the nearby surface, and thus generating the reactive species leading to efficient surface reactions. It is, thus, obvious that nanostructures offer the opportunity for the charges to survive in order to induce a surface reaction, before recombination occurs.

As well as scientific research, engineered systems such as solar water pumping systems or solar dryers highlighted the benefit of solar powered applications that could be implemented already today.

The conference highlights were not only scientific, but also cultural and culinary. A full day was dedicated to visit the West Bank of Luxor, including memorable visits to the Valley of the Kings, the mortuary temple of pharaoh Hatshepsut, and Hapu Temple of Ramsis 3rd. Well preserved colour reliefs, built 4000 years ago, were simply breath-taking and demonstrated that they were built to last for eternity. Today we have lost the desire to build for eternity; instead, we are burning up so much fossil fuel that it is doubtful whether there will be anything left for future generations. Power from the sun can be harnessed to provide sustainable energy, and many solar-based technologies are ready to be implemented now.

The highly successful solar conference series, started in 1991, provide a place where researchers interested in fundamental and applied aspects of photochemistry can meet and inspire one another. The solar conferences recognize that successful photochemical applications go hand in hand with advancement of fundamental understanding of photoinduced processes and excited states.

The focus of this conference series is of great importance to the current global energy security situation. A coordinated action on a global level is urgently needed to avert the crisis. The developing world must take an active role in any such coordinated action.
Currently, nanotechnology is generating a lot of attention and it is generating great expectations not only in the academic community but also among investors, the governments, and industry. Its unique capability to fabricate new structures at atomic scale has already produced novel materials and devices with great potential applications in a wide number of fields. Among them, significant breakthroughs are especially required in the energy sector that will allow us to maintain our increasing demand for energy. Nanotechnology offers, for the first time, tools to develop new industries based on cost-effective and cost-efficient economies, thus seriously contributing to a sustainable economic growth.

This special issue covers some specific contributions from nanotechnology to various sustainable energies. As mentioned above, the issue's main focus is on three broad areas, namely, light harvesting, catalysis, and materials. Articles on PV chemical solar cells are the most significant examples of the contributions of nanotechnology in the energy sector. The aim of this issue is to present some significant contributions from different research groups working on different approaches, to find solutions to one of the great challenges of our time, that is, the production and use of greener energy from one of the most exciting and multidisciplinary fields, nanotechnology.

Focusing on the energy domain, nanotechnology has the potential to significantly reduce the impact of energy production, storage, and use. Even if we are still far from a truly sustainable energy system, the scientific community is looking at a further development of energy nanotechnologies. In fact, one of the 10 top-level themes of the VII Framework Program of the European Union (FP7) is energy. Accordingly, the research will be focused on accelerating the development of cost-effective technologies for a more sustainable energy economy.

According to the "Roadmap Report Concerning the use of Nanomaterials in the Energy Sector" from the 6th Framework Program, the most promising application fields for the energy conversion domain will be mainly focused on solar energy (mostly photovoltaic technology for local supply), hydrogen conversion, and thermoelectric devices.

It is hoped that this issue may provide an overview of the contribution of nanotechnology to the solar energy in a broad sense and to sustainable ways to store energy as a step forward a more sustainable use of energy.

M. S. A. Abdel-Mottaleb Frank Nüesch Mohamed M. S. A. Abdel-Mottaleb 


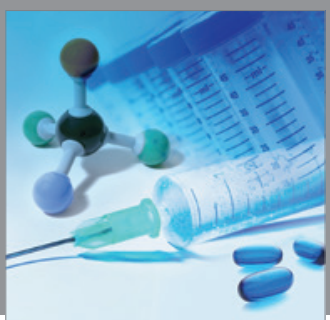

International Journal of

Medicinal Chemistry

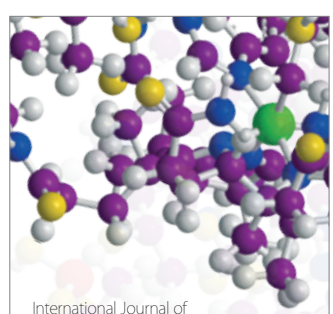

Carbohydrate Chemistry

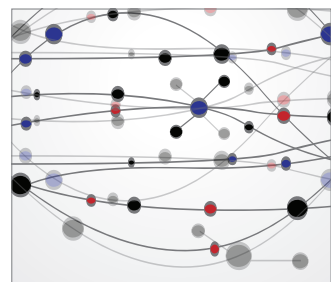

The Scientific World Journal
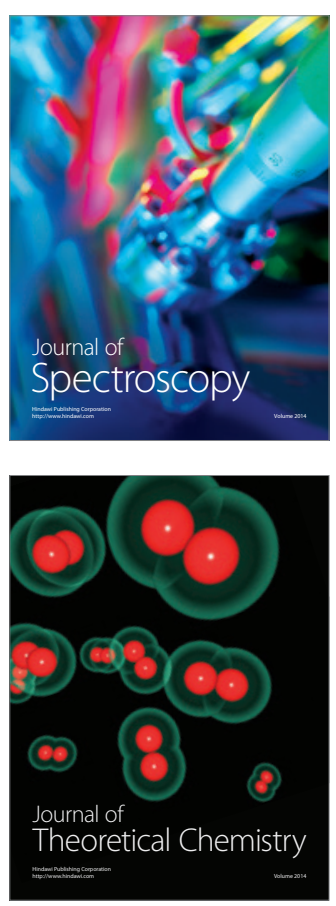
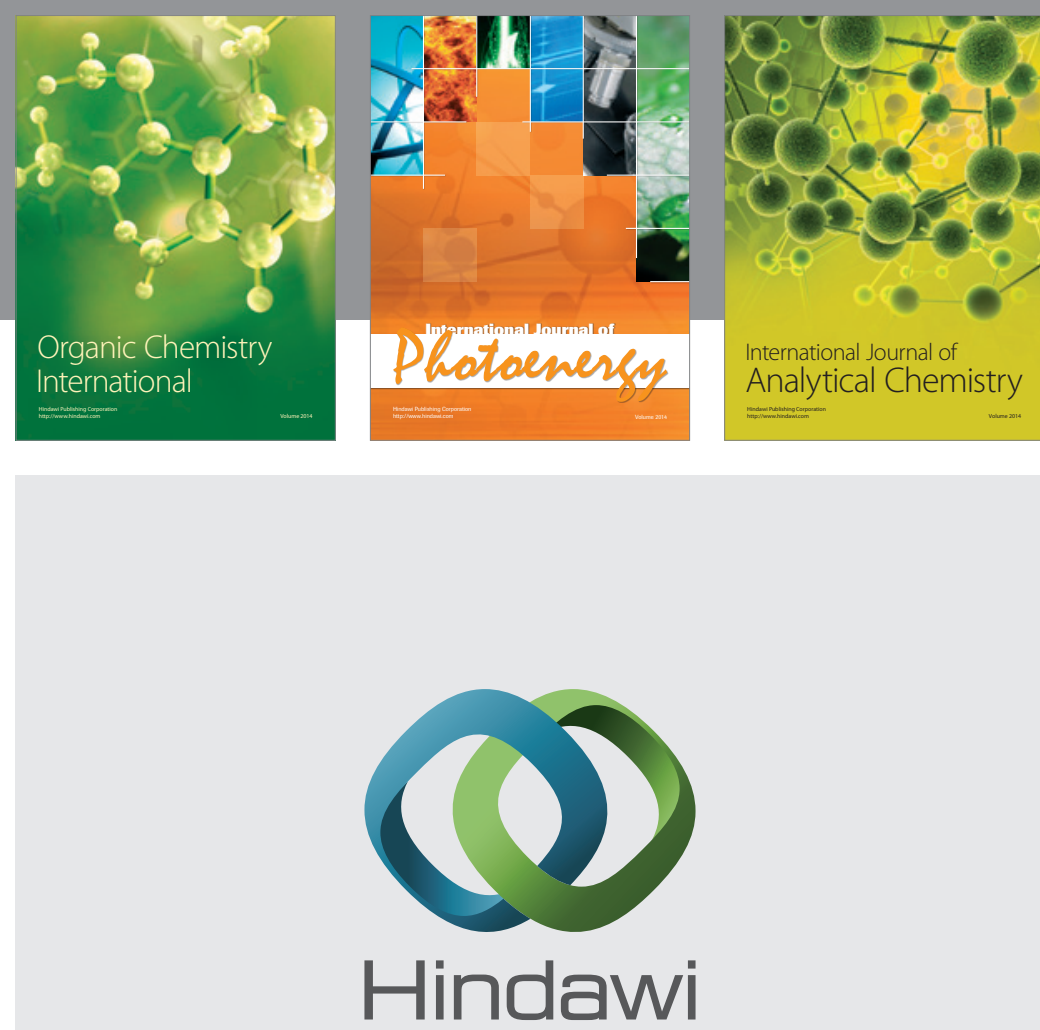

Submit your manuscripts at

http://www.hindawi.com
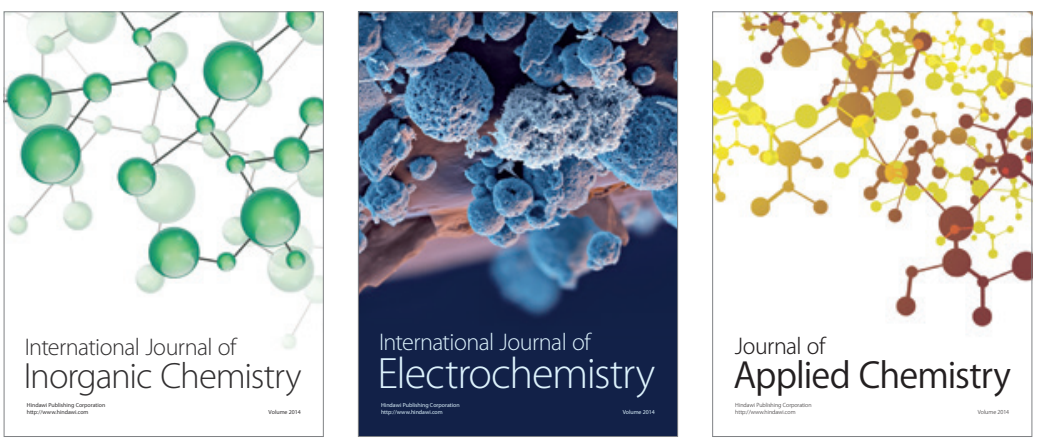

Journal of

Applied Chemistry
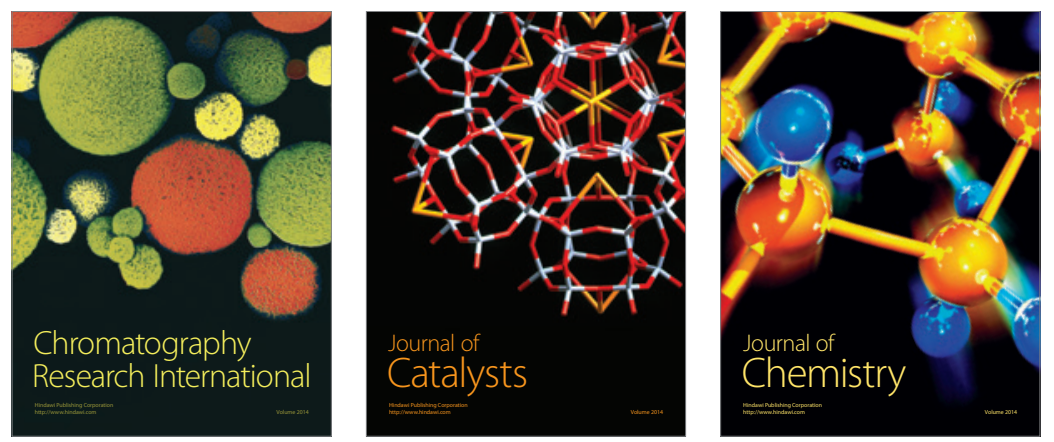
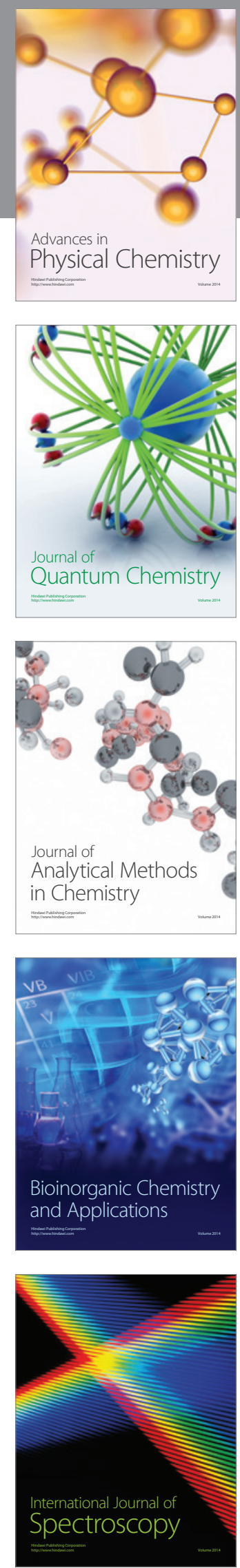